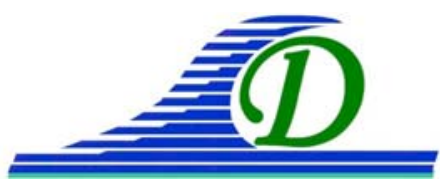
XIII ${ }^{\text {èmes }}$ Journées Nationales Génie Côtier - Génie Civil
Dunkerque, 2-4 juillet 2014

DOI:10.5150/jngcgc.2014.083 (c) Editions Paralia CFL

disponible en ligne - $h t t p: / / w w w . p a r a l i a . f r$ - available online

\title{
Simulation numérique du phénomène de franchissement d'ouvrage par une houle irrégulière
}

\author{
Cyril MOKRANI ${ }^{1}$, Stéphane ABADIE ${ }^{1}$, Pierre LUBIN $^{2}$
}

1. SIAME, EA4581, Université de Pau et des Pays de l'Adour, Allée du parc Montaury, 64600 Anglet, France. cyril.mokrani@gmail.com ; stephane.abadie@univ-pau.fr

2. I2M, UMR 5295, Université de Bordeaux, F-33400 Talence, France.

p.lubin@i2m.u-bordeaux1.fr

\section{Résumé :}

Dans le cadre du projet EMACOP (Énergie MArine CÔtière et Portuaire, http://www.emacop.fr/), une étude numérique est réalisée afin de tester la validité d'un modèle Navier-Stokes/VOF pour la simulation du processus de franchissement intervenant sur des systèmes de récupération d'énergie marine. Dans l'article, nous présentons la méthode numérique utilisée pour générer une houle irrégulière, puis nous étudions différents cas de franchissement d'un obstacle incliné par une houle irrégulière. Les volumes de franchissement simulés sont comparés aux mesures expérimentales disponibles dans la littérature.

Mots-clés : Simulation Navier-Stokes, Débits de franchissement, Energie de la houle, Houle irrégulière.

\section{Introduction}

Le projet national Énergie MArine CÔtière et Portuaire (EMACOP, http://www.emacop.fr/) a pour objectif la caractérisation du potentiel énergétique exploitable au voisinage des ouvrages côtiers et portuaires et l'évaluation des possibilités de couplage des techniques actuelles aux ouvrages afin de récupérer cette énergie. Dans EMACOP on cherche également à évaluer la précision et l'adéquation des modèles numériques existants pour contribuer à atteindre les objectifs du programme. Le système à franchissement Sea Slot-cone Generator (SSG, e.g., MARGHERITINI et al., 2009) figure parmi les systèmes étudiés au sein du projet. L'objectif de cette communication est de tester la validité d'un modèle Navier-Stokes/VOF pour simuler le franchissement et donc à terme la faisabilité de calculs de rendement de systèmes SSG à l'aide de ce type de modèle. 


\section{Présentation du modèle numérique}

\subsection{Le code THETIS}

Le modèle numérique utilisé dans cet article est le code THETIS (http://thetis.enscbp.fr/) développé à l'Institut de Mécanique et d’Ingénierie de Bordeaux. Ce code résout (entre autre) les équations de Navier-Stokes incompressibles sur maillages fixes pour un écoulement comportant plusieurs phases (dans le cas présent : l'eau et l'air) en formulation 1-fluide. La distinction entre les phases se fait dans les paramètres physiques qui apparaissent dans les équations de Navier-Stokes (i.e. la viscosité $\mu$ et la masse volumique $\rho$ ). Ces équations sont exactes sauf dans les mailles contenant les interfaces où les propriétés du mélange sont seulement approchées. La résolution numérique des équations de l'écoulement repose ensuite sur une discrétisation en volume fini sur un maillage cartésien et un découplage vitesse-pression par une méthode de projection. A chaque itération temps, l'interface entre les phases est capturée et déplacée suivant la méthode VOF-TVD (LUBIN et al., 2006), qui permet de limiter la diffusion numérique lors de la résolution de l'équation d'advection de l'interface. Les obstacles, tels qu'une digue par exemple, sont modélisés par la méthode des domaines fictifs associée à une pénalisation par perméabilité. D'un point de vue physique, cela revient à considérer que le solide est un milieu poreux à perméabilité nulle, qui ne laisse donc pas passer le fluide. D'un point de vue numérique, on pénalise la perméabilité du fluide $K$ sur la partie du domaine que l'on souhaite rendre solide via un terme supplémentaire introduit dans l'équation de quantité de mouvement de NavierStokes (BRINKMAN, 1947). Des zones réellement poreuses peuvent également être introduite dans le calcul par exemple pour absorber la houle. De plus amples informations sur ce modèle et des applications sur des problèmes liés aux vagues pourront être trouvées par exemple dans ABADIE et al. (2010) ou LUBIN et al. (2006 $\& 2011)$.

\subsection{Générateur de houle irrégulière}

Le batteur implémenté dans le code THETIS génère la houle à partir d'un terme source local $S(x, y, t)$ ajouté à l'équation de conservation de la masse (LIN \& LIU, 1999) :

$$
\frac{\partial u}{\partial x}+\frac{\partial v}{\partial y}=S(x, y, t)
$$

Ce terme qui contrôle l'apport de masse dans le milieu, permet de produire différents type d'ondes de surface (e.g., houles de Stokes jusqu'à l'ordre 5, ondes cnoïdales et ondes solitaires) suivant sa forme analytique. LUBIN et al. (2011) propose un exemple d'utilisation de ce batteur avec THETIS pour l'étude du déferlement sur une plage à pente constante. 


\section{XIII ${ }^{\text {èmes }}$ Journées Nationales Génie Côtier - Génie Civil \\ Dunkerque, 2-4 juillet 2014}

Le terme source a, dans un premier temps, été modifié de façon à pouvoir générer une houle irrégulière suivant la paramétrisation de JONSWAP (HASSELMANN et al., 1973) qui est utilisée dans la plupart des tests expérimentaux mesurant les débits de franchissement. Un spectre de JONSWAP est caractérisé par une densité d'énergie spectrale associée à chaque fréquence qui s'écrit sous la forme suivante :

$$
S(\omega)=\alpha \gamma^{a} H_{S}^{2} \omega_{0}^{4} \omega^{-5} e^{\frac{-5}{4}\left(\frac{\omega_{0}}{\omega}\right)^{4}} \text { avec a }=\mathrm{e}^{\frac{\left(\omega-\omega_{O}\right)^{2}}{2 \sigma^{2} \omega_{0}^{2}}}
$$

$H_{s}$ et $\omega_{0}$ représentent respectivement la hauteur significative et la pulsation au pic. Dans cette étude, le paramètre $\gamma$ est fixé égal à 3.3 et le coefficient $\sigma$ à 0.1 pour $\omega<\omega_{0}$ et 0.5 pour $\omega>\omega_{0}$. Le coefficient $\alpha$ est à ajuster pour chaque état de mer pour normaliser le spectre. Il est fixé de façon à respecter la relation :

$$
H_{s}^{2}=16 \int S(\omega) \mathrm{d} \omega
$$

Le spectre que l'on souhaite modéliser est tout d'abord discrétisé en un nombre de raies $N_{r}$. Puis on associe à chaque pulsation une densité d'énergie spectrale à laquelle correspond une amplitude. Le nombre d'onde correspondant à chaque bande se déduit ensuite en utilisant la relation de dispersion. Un déphasage aléatoire est enfin imposé sur chaque bande. Finalement, le signal de surface prend la forme :

$$
h(\mathrm{x}, \mathrm{t})=\sum a_{i} \sin \left(\omega_{i} t-k_{i} \mathrm{x}+\phi_{i}\right)
$$

auquel on peut facilement associer le terme source correspondant (LIN \& LIU, 1999).

Afin de tester l'implémentation de ce batteur de houle irrégulière, nous avons réalisé des simulations dans le domaine représenté en figure 1. Ce dernier fait 22 mètres de long et 0.5 mètres de haut. La source de masse générant les vagues est placée au milieu du domaine de profondeur $h_{0}=0.2 \mathrm{~m}$. Deux zones poreuses dissipatives (porosité variant linéairement pour une dissipation graduelle) sont placées à proximité des limites gauche et droite du domaine. Les conditions limites latérales permettent la sortie ou l'entrée du delta de masse associé à la source.

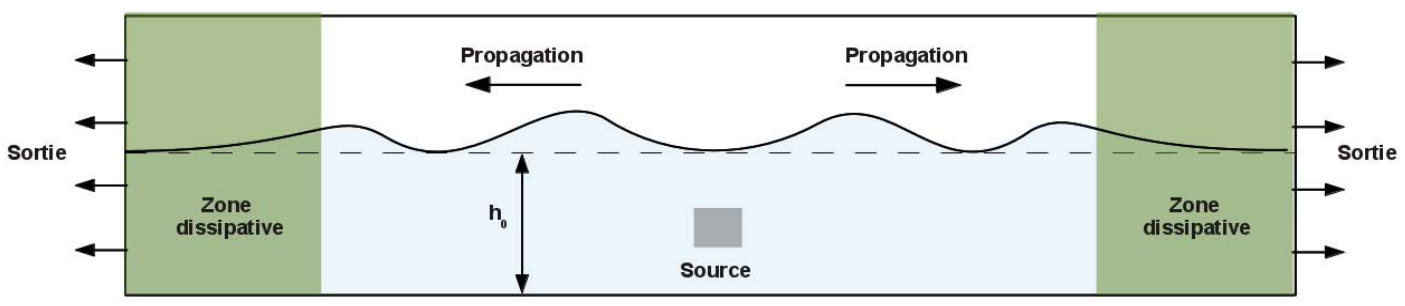

Figure 1. Schématisation du domaine de calcul pour la validation du générateur de houle irrégulière.

Le choix du maillage est important. En effet, nos calculs ont montré que pour des maillages trop grossiers, la simulation de la houle régulière posait déjà des problèmes 


\section{Thème 5 - Énergies et ressources marines}

importants avec l'apparition d'ondes basses fréquences pouvant faire diverger le calcul. D'un autre côté, la simulation d'une houle spectrale nécessite un calcul sur un nombre important de périodes pour être représentatif et l'on est vite confronté à la limite des ressources de calcul. Le compromis auquel nous sommes arrivés est un maillage irrégulier dans les deux directions. Le maillage vertical est plus fin à proximité de l'interface $\left(\Delta Z_{\min }=0.005 \mathrm{~m}\right)$ et le maillage horizontal au centre du domaine $\left(\Delta X_{\min }=0.12 \mathrm{~m}\right)$. Cette configuration correspond à 100 cellules dans les deux directions et permet de calculer 100 périodes de vague en environ 20 minutes CPU sur un seul processeur.

Les paramètres caractéristiques du spectre de JONSWAP simulé sont $H_{\mathrm{s}}=0.04 \mathrm{~m}$ et $T_{p}=0.566 \mathrm{~s}$. Il est discrétisé en utilisant 1000 raies. Afin de valider le batteur à houle irrégulière, nous avons comparé le spectre simulé à $5 \mathrm{~m}$ de la source avec le spectre que nous cherchons à modéliser. Pour cela, nous avons dans un premier temps enregistré le signal de surface libre au point choisi puis calculé la transformée de Fourier. Le résultat obtenu est comparé avec le spectre de JONSWAP théorique en figure 2. L'allure générale du spectre est globalement bien reproduite. Le zoom permet néanmoins de voir que le spectre simulé est légèrement moins énergétique que le spectre initial même si les amplitudes correspondantes à certaines fréquences sont au contraire surestimées. Cette très légèrement diminution d'énergie peut s'expliquer par la diffusion numérique ( $a$ priori d'autant plus importante que la distance de propagation est longue).

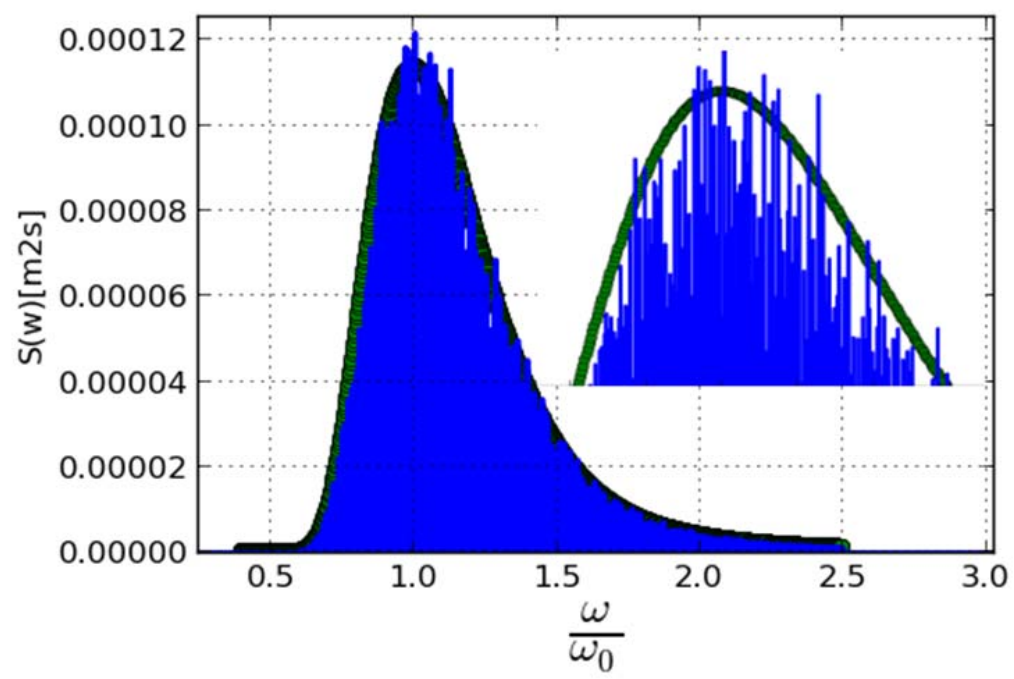

Figure 2. Comparaison entre le spectre théorique de JONSWAP théorique (oo) et le spectre simulé à $5 \mathrm{~m}$ de la source obtenu par transformée de Fourier discrète du signal (raies bleues). 


\section{XIII ${ }^{\text {èmes }}$ Journées Nationales Génie Côtier - Génie Civil \\ Dunkerque, 2-4 juillet 2014}

\section{Simulation du franchissement d'ouvrage}

Dans cette partie, nous étudions les capacités du modèle à reproduire le phénomène de franchissement d'ouvrage en houle irrégulière en comparant les simulations aux tests expérimentaux réalisés par KOFOED (2002). Le cas simulé correspondant à l'expérience est schématisé en figure 3. Les expériences présentées dans KOFOED (2002) ont été réalisées à échelle réduite avec un facteur d'échelle de 1:50 ${ }^{\mathrm{ème}}$. La figure 3 représente l'expérience considérée. Les paramètres de l'expérience sont: $h_{0}=0.5 \mathrm{~m}$ (soit $25 \mathrm{~m}$ à échelle réelle), $d_{r}=0.32 h_{0}$ (i.e., $d_{r}=0.162 \mathrm{~m}$ ), $R_{c}=0.16 h_{0}$ (i.e., $0.077 \mathrm{~m}$ ) et une pente d'ouvrage de $20^{\circ}$. Les débits de franchissement sont calculés à l'abscisse $X_{0}=5.32 h_{0}$ (i.e., $2.66 \mathrm{~m}$ ). Les essais ont duré trente minutes, ce qui représente $3.5 h$ à l'échelle réelle.

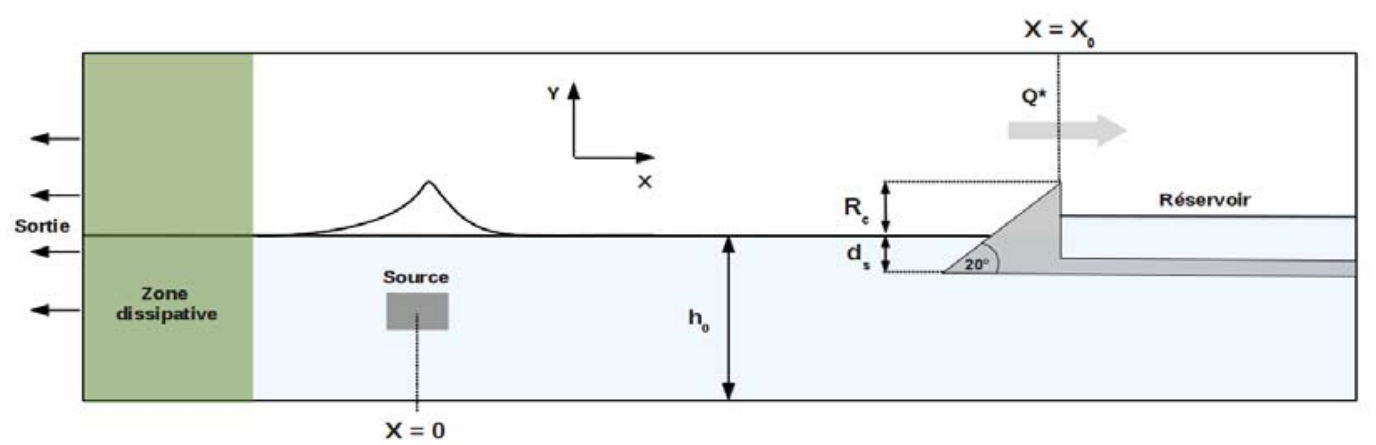

Figure 3. Schématisation du domaine de calcul pour le cas de franchissement d'ouvrage en houle irrégulière.

Tableau 1. Caractéristiques des houles irrégulières testées lors les essais de KOFOED (2002).

\begin{tabular}{lllllllllll}
\hline Cas & $\mathbf{1}$ & $\mathbf{2}$ & $\mathbf{3}$ & $\mathbf{4}$ & $\mathbf{5}$ & $\mathbf{6}$ & $\mathbf{7}$ & $\mathbf{8}$ & $\mathbf{9}$ & $\mathbf{1 0}$ \\
\hline $\boldsymbol{T}_{\boldsymbol{p}}(\mathbf{s})$ & 0.566 & 0.849 & 0.849 & 1.131 & 1.131 & 1.131 & 1.414 & 1.697 & 1.697 & 1.131 \\
$\boldsymbol{H}_{\boldsymbol{s}}(\boldsymbol{m})$ & 0.04 & 0.08 & 0.10 & 0.12 & 0.14 & 0.16 & 0.16 & 0.12 & 0.16 & 0.04 \\
$\boldsymbol{R}_{\boldsymbol{c}}{ }^{*}$ & 2.00 & 1.00 & 0.80 & 0.64 & 0.55 & 0.50 & 0.48 & 0.64 & 2.00 & 2.00 \\
$\boldsymbol{\xi}_{0 p}$ & 1.3 & 1.4 & 1.2 & 1.5 & 1.4 & 1.3 & 1.6 & 2.2 & 1.9 & 2.6 \\
\hline
\end{tabular}

$R_{c}{ }^{*}$ est la revanche adimensionnelle (i.e., $\left.R c / H s\right)$ et $\xi_{o p}$ le paramètre d'Iribarren

Parmi ces essais, nous avons considéré 10 cas de houles irrégulières qui correspondent à différents nombres d'Iribarren (cf. tableau 1), estimés en fonction de l'angle $\alpha$ du talus de la structure, de la période pic, et de la hauteur significative selon la formule :

$\xi_{0 \mathrm{p}}=\frac{\tan (\alpha)}{\sqrt{s_{0 \mathrm{p}}}}$ avec $s_{0 \mathrm{p}}=\frac{H_{s}}{L_{0 \mathrm{p}}}$ et $L_{0 \mathrm{p}}=\frac{\mathrm{gT}_{p}^{2}}{2 \pi}$

La figure 4 présente différents iso-contours de la fonction couleur $F$ (qui représente le taux de présence en eau dans chaque cellule) durant le processus de franchissement 


\section{Thème 5 - Énergies et ressources marines}

correspondant au cas 5 qui implique un déferlement plongeant nette sur le talus. Cette figure permet de visualiser la forme de la vague (iso-contour $F=0.5$ ), ainsi que la distribution eau/air pendant le déferlement (figure 4-a et 4-b), le processus de franchissement (figure 4-c, 4-d) et de retrait (figure 4-e). Ces résultats ne peuvent en revanche pas être comparés à l'expérience qui s'est concentrée sur la mesure de débits de franchissement.

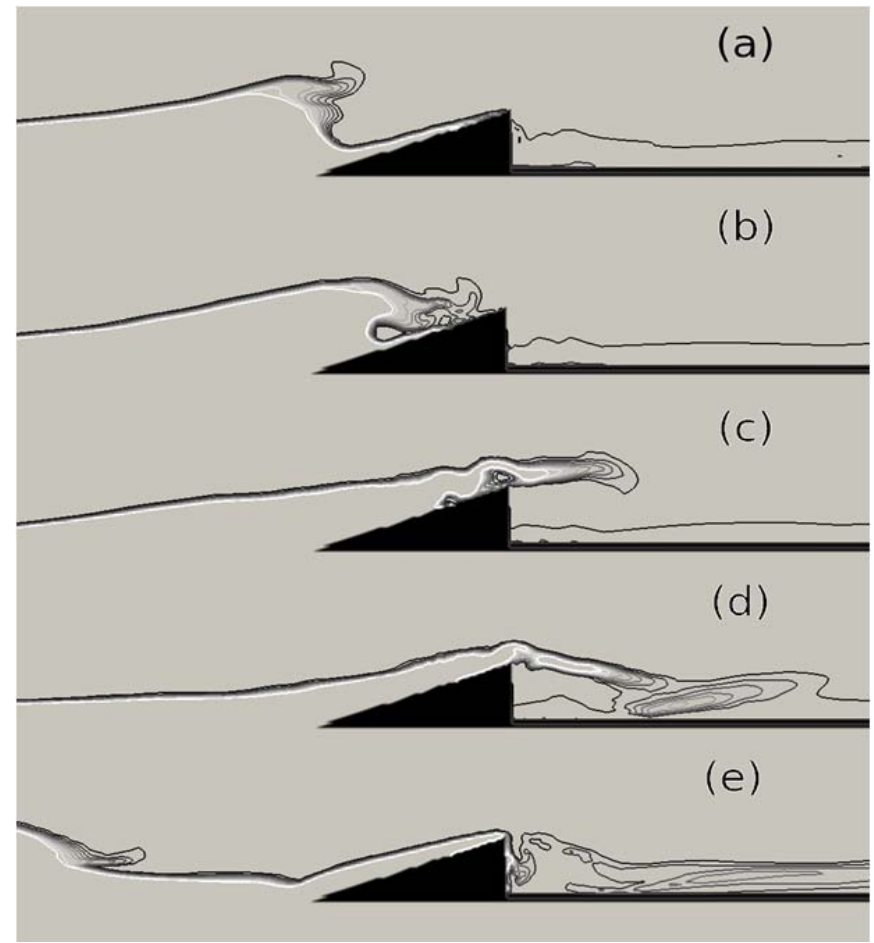

Figure 4. Détail de la séquence de franchissement pour un cas de déferlement plongeant (cas 5, tableau 1). Iso contours de la fraction volumique d'eau F, du plus foncé au plus clair : $F=0$, 0.0625, 0.125, 0.1875, 0.250, 0.312, 0.375, 0.4375, 0.500. (a) $t=10.2 \mathrm{~s}$, (b) $t=10.4 \mathrm{~s}$, (c) $t=10.6 \mathrm{~s}$, (d) $t=10.9 \mathrm{~s}$, (e) $t=11.2 \mathrm{~s}$. A noter que l'iso contour $F=0.5$ représente la position de l'interface.

Dans le cadre de cette étude, et pour des raisons de réduction du temps de calcul, nous n'avons simulé qu'une partie de l'expérience. En effet, KOFOED (2002) fournit des mesures de débits de franchissement moyennées sur 30 minutes. Les calculs présentés dans notre étude ont été réalisés en séquentiel (i.e. sur un seul processeur) car certaines routines développées spécifiquement dans le cadre d'EMACOP n'ont pas encore été parallélisées. Une simulation de 30 minutes sur un seul processeur durerait plusieurs dizaines de jours ce qui nous a semblé rédhibitoire. (A court terme, ce type de calcul pourra aisément être réalisé en moins de 5 jours en utilisant une dizaine de processeurs. 


\section{XIII ${ }^{\text {èmes }}$ Journées Nationales Génie Côtier - Génie Civil \\ Dunkerque, 2-4 juillet 2014}

Le code THETIS est en effet entièrement parallélisé et le travail restant a effectuer pour paralléliser les routines récentes est relativement réduit).

Simuler uniquement le début de l'expérience peut se justifier par le fait que la moyenne du débit de franchissement n'évolue plus que faiblement au-delà d'une certaine durée assez courte. Ainsi, pour tous les cas considérés, la valeur moyenne du débit adimensionnel $Q^{*}\left(Q^{*}=Q / Q_{0}\right.$ où $\left.Q_{0}=H_{0}\left(g H_{0}\right)^{1 / 2}\right)$ semble se stabiliser après un durée proche de $t \sim 120 \mathrm{sec}$, et finalement tendre vers une valeur limite. La figure 5 montre en exemple la variation temporelle de la moyenne correspondant au cas 5. Pour cette raison, l'ensemble de nos calculs ont été effectués sur 250 s (soit suivant les cas de $~ 150$ à 500 vagues simulées), ce qui a permis de réduire le temps CPU de chaque simulation à environ $48 \mathrm{~h}$.

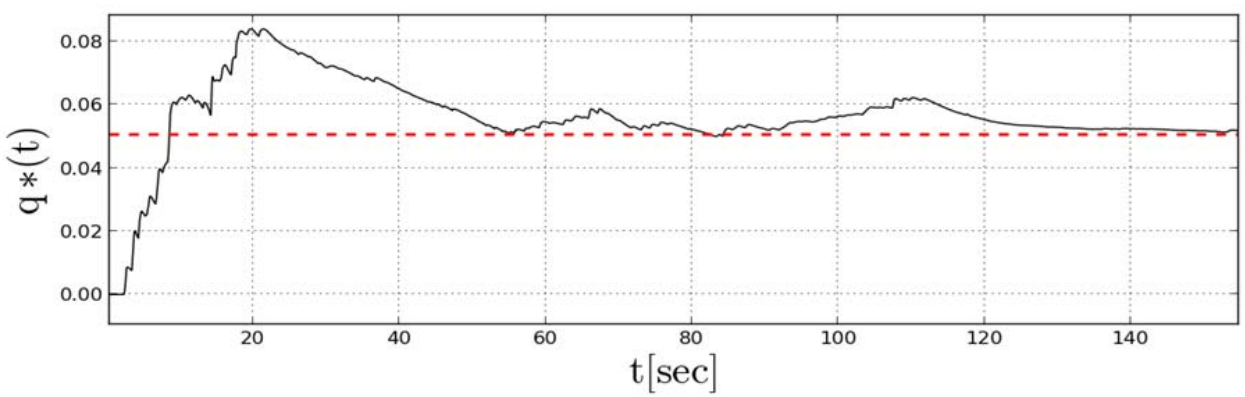

Figure 5. (-): Évolution temporelle de la moyenne du débit adimensionnel de franchissement (cas 5) et (--) : valeur moyenne utilisée pour la comparaison avec la valeur expérimentale issue de KOFOED (2002).

La figure 6 présente les valeurs de débits adimensionnels moyennées pour chaque cas considéré en fonction de la revanche adimensionnelle $R_{c}{ }^{*}$ (i.e., hauteur de l'obstacle $R_{c}$ divisée par $H_{s}$ ). Nous avons également ajouté sur ce graphe les valeurs de débit de franchissement obtenus à l'aide de la formule suivante (VAN DER MEER \& JANSSEN, 1995) :

$Q^{*}=\frac{0.06}{\tan (\alpha)} \xi_{0 p} \exp \left(-4.7 \frac{R_{C}}{\xi_{0 p}}\right)$, pour: $\xi_{0 p}<2$

Cette formule a été utilisée pour calculer les points en vert sur la figure 6 dans les cas où le critère $\xi_{0 p}<2$ est vérifié. Nous avons enfin tenté d'ajuster une loi exponentielle du type $Q^{*}=\mathrm{a} \mathrm{e}^{b R_{c}}$ sur les données de simulations et les données expérimentales.

Tout d'abord, on remarque que les résultats obtenus par le code THETIS sont relativement proches des valeurs expérimentales de Kofoed et la tendance globale des résultats est globalement respectée. La dépendance au nombre d'Iribarren explique la présence de plusieurs points pour chaque jeu de données pour une même revanche adimensionnelle. L'ajustement par la loi exponentielle sur les données de KOFOED 
(2002) donnent les valeurs suivantes : $a=0.08$ et $b=-1.95$, indépendamment du paramètre d'Iribarren $\xi_{\text {op }}$. Les simulations THETIS donnent également des quantités de franchissement faiblement dépendantes de $\xi_{0 p}$, avec des valeurs de $\mathrm{a}=0.125$ et $\mathrm{b}=-1.975$. Cela donne une tendance très proche de la loi empirique proposée par VAN DER MEER \& JANSSEN (1995) ce qui est prometteur.

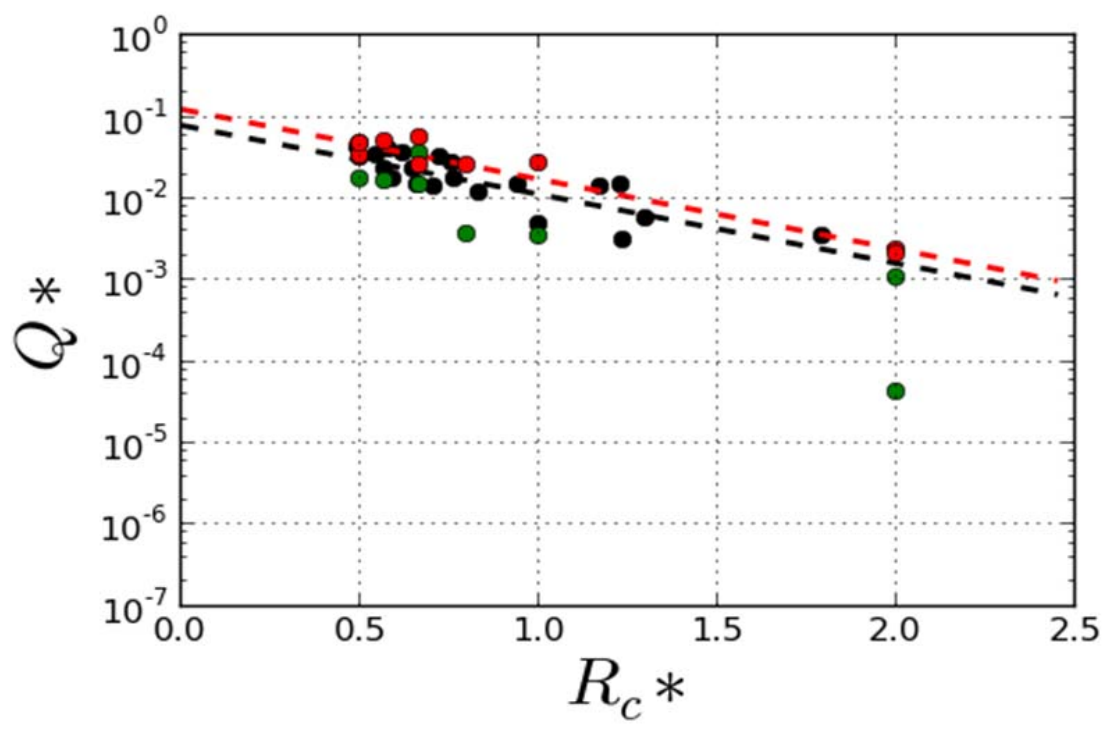

Figure 6. Valeurs des débits de franchissement moyennés en fonction de la revanche adimensionnelle Rc* - (o) :THETIS , (o): Valeurs expérimentales (KOFOED, 2002),

(o) Formule de VAN DER MEER \& JANSSEN (1995), (---) : ajustement par loi exponentielle sur les données issues de KOFOED (2002), (---) : ajustement par loi exponentielle sur les résultats THETIS.

\section{Conclusions}

Dans cet article, nous avons réalisé des simulations de franchissement d'ouvrage en houle irrégulière à l'aide du code Navier-Stokes THETIS. Les valeurs de débits moyens obtenus correspondent bien aux mesures expérimentales et montrent que ce type de modèle est une alternative intéressante à la mesure. En termes de temps de calcul, un spectre complet doit pouvoir être réalisé en 1 à 5 jours CPU (en fonction de la résolution) en version parallèle.

\section{Remerciements}

Ce travail a reçu le soutien financier du programme national EMACOP. 


\section{XIII ${ }^{\text {èmes }}$ Journées Nationales Génie Côtier - Génie Civil \\ Dunkerque, 2-4 juillet 2014}

\section{Références bibliographiques}

ABADIE S, MORICHON D., GRILLI S., GLOCKNER S. (2010). Numerical Simulation of waves generated by landslides using a multiple-fluid Navier-Stokes model. Coastal Engineering, Vol. 57, pp 779-794. http://dx.doi.org/10.1016/j.coastaleng.2010.03.003

BRINKMAN H.C. (1947). A calculation of the viscous force exerted by a flowing fluid on a dense swarm of particles. Applied Scientific Research Section a-Mechanics Heat Chemical Engineering Mathematical Methods, Vol. 1(1), pp 27-34.

HASSELMANN K., BARNETT T.P., BOUWS E., CARLSON H., CARTWRIGHT D.E., ENKE K., EWING J.A., GIENAPP H., HASSELMANN D.E., KRUSEMAN P., MEERBURG A.J., MÜLLER P., OLBERS D.J., RICHTER K., SELL W., WALDEN H. (1973). Measurements of wind-wave growth and swell decay during the Joint North Sea Wave Project (JONSWAP). Deutsches Hydrographisches Intitut- Hamburg.

KOFOED J.P. (2002). Wave Overtopping of Marine Structures - Utilization of Wave Energy. Ph. D. Thesis. Hydraulic and Coastal Engineering Laboratory, Department of Civil Engineering, Aalborg University, Series Paper No. 24.

LIN P., LIU P.L.-F. (1999). Internal Wave-Maker for Navier-Stokes equations Models. Journal of Waterways, Port, Coastal and Ocean Engineering. Vol. 125(4), pp 207-215. http://dx.doi.org/10.1061/(ASCE)0733-950X(1999)125:4(207)

LUBIN P., VINCENT S., ABADIE S., CALTAGIRONE J.-P. (2006). Threedimensional Large Eddy Simulation of air entrainment under plunging breaking waves. Coastal Engineering, Vol. 53, pp. 631-655. http://dx.doi.org/10.1016/j.coastaleng.2006.01.001 LUBIN P., GLOCKNER S., KIMMOUN O., BRANGER H. (2011). Numerical study of the hydrodynamics of regular wave breaking over a sloping beach. European Journal of Mechanics - B/Fluids, Vol. 30(6), pp 552-564. http://dx.doi.org/10.1016/j.euromechflu.2011.01.001 MARGHERITINI L., VICINANZA D., FRIGAARD P. (2009). SSG wave energy converter: Design, reliability and hydraulic performance of an innovative overtopping device. Renewable Energy, Vol. 34(5), pp 1371-1380. http://dx.doi.org/10.1016/j.renene.2008.09.009 VAN DER MEER J.W., JANSSEN J.P.F.M. (1995). Wave run-up and wave overtopping at dikes. In : wave forces on inclined and vertical wall structure, ASCE. Ed. N.,Kobayashi and Z. Demibilek, Chap 1, pp 1-27. 
Thème 5 - Énergies et ressources marines 